\title{
Autotuning of Isotropic Hardening Constitutive Models on Real Steel Buckling Data with Finite Element Based Multistart Global Optimisation on Parallel Computers
}

\author{
Anton Shterenlikht, ${ }^{1}$ Mohammad M. Kashani, ${ }^{1}$ Nicholas A. Alexander, ${ }^{1}$ \\ Gethin Williams, ${ }^{2}$ and Adam J. Crewe ${ }^{1}$ \\ ${ }^{1}$ Faculty of Engineering, The University of Bristol, Bristol BS8 1TR, UK \\ ${ }^{2}$ Advanced Computing Research Centre, The University of Bristol, Bristol BS8 1QU, UK \\ Correspondence should be addressed to Anton Shterenlikht; mexas@bris.ac.uk
}

Received 9 August 2016; Revised 18 October 2016; Accepted 6 December 2016; Published 16 January 2017

Academic Editor: Jean-Michel Bergheau

Copyright (C) 2017 Anton Shterenlikht et al. This is an open access article distributed under the Creative Commons Attribution License, which permits unrestricted use, distribution, and reproduction in any medium, provided the original work is properly cited.

\begin{abstract}
An automatic framework for tuning plastic constitutive models is proposed. It is based on multistart global optimisation method, where the objective function is provided by the results of multiple elastoplastic finite element analyses, executed concurrently. Wrapper scripts were developed for fully automatic preprocessing, including model and mesh generation, analysis, and postprocessing. The framework is applied to an isotropic power hardening plasticity using real load/displacement data from multiple steel buckling tests. M. J. D. Powell's BOBYQA constrained optimisation package was used for local optimisation. It is shown that using the real data presents multiple problems to the optimisation process because (1) the objective function can be discontinuous, yet (2) relatively flat around multiple local minima, with (3) similar values of the objective function for different local minima. As a consequence the estimate of the global minimum is sensitive to the amount of experimental data and experimental noise. The framework includes the verification step, where the estimate of the global minimum is verified on a different geometry and loading. A tensile test was used for verification in this work. The speed of the method critically depends on the ability to effectively parallelise the finite element solver. Three levels of parallelisation were exploited in this work. The ultimate limitation was the availability of the finite element commercial solver license tokens.
\end{abstract}

\section{Introduction}

Experimentally validated constitutive models are one of the most important components of nonlinear finite element (FE) analyses, especially if the goal is accurate and robust system performance predictions to a range of different loading scenarios. However, obtaining robust and optimally calibrated constitutive models from historic, experimental tests can require the solution of a large number of nonlinear FE analyses which may be prohibitively computationally expensive. A complex nonlinear inverse problem needs to be solved. This inverse problem can be recast as a nonlinear multidimensional optimisation of objective function $f$. Note that $f$ may well contain problems associated with many local optima and discontinuities. In this paper we present a generic and rigorous framework for exploring this problem automatically with parallel distributed computation.

As a test case we seek to obtain the nonlinear constitutive model of steel reinforcing bars from a limited set of real, historic bar buckling tests of different geometries [1-5]. The general context of the buckling of vertical reinforcement is that it is the most common type of observed failure mechanism in concrete columns subject to earthquake loading [6-8]. This example highlights a number of difficulties with adopting an ad hoc approach rather than a rigorous and generic one based on nonlinear optimisers, such as line search or trust region methods $[9,10]$. These complicating factors include (i) low gradients of $f$, (ii) many local optima of $f$, and (iii) a loading arrangement and boundary conditions which can result in discontinuities of $f$. 
In this paper the parameters of an isotropic power hardening plastic model are tuned using elastoplastic buckling tests of reinforcing bars. The only experimental data used in this work were the load-displacement traces from five buckling tests. All rods had the same diameter, $d=12 \mathrm{~mm}$, and different lengths, $l$. The bars are identified by their $l / d$ ratios, $5,8,10,15$, and $20[1,2,5]$.

Various inverse techniques for identification of material parameters have been investigated in recent years [11]. Although point measurement approaches (strain gauges) can deliver useful identifications, even at elevated temperatures [12], most new methods grew from rapid advances in full field experimental methods, in particular the digital image correlation (DIC) technique. Accordingly most of the published inverse methods rely on specially designed specimens, which exhibit the widest variability of stress-strain histories and/or stress triaxialities and which supply ample displacement or strain data via DIC measurements [13]. In cases where neither is available, some of the methods, for example, the virtual fields method, cannot be used effectively. Moreover, some methods are restricted by the assumption of elasticity, for example, the constitutive equation gap method, the equilibrium gap method [11], or the elastodynamics experimental approach $[14,15]$. Some other inverse methods are specific to optimisation of elastoplastic properties during the manufacturing process design stage [16].

All engineering inverse methods are a subset of the simulation-based optimisation research area [17, 18]. In common with other simulation-based optimisation problems $[19,20]$, this work highlights two major problems: (i) the evaluation of objective functions is costly and (ii) the form of the objective function is unknown. Effective parallelisation and full automation are the key to successful simulationbased global optimisation. Multistart optimisation [21-23] is a good choice for parallel distributed computers. The choice of starting points, for the global optimiser, is taken at random or at predetermined intervals. Therefore the choice of the subsequent starting points does not depend on the local minima, $\mathbf{x}^{*}$, calculated from previous starting points. In such cases no interprocess communication is involved, and multiple local optimisers run completely independently. The main computational advantage is that the time of investigating many starting points is the same as for a single point. In principle, the extent of parallelisation is limited by the size of the computer.

In practice, other factors limit parallel scaling, for example, licensing restrictions on running multiple concurrent instances of commercial FE software. In this work multiple local searches are used to find the estimate of the global optimum independently. This is in contrast to the most popular hybrid global optimisation methods, where local search is used only to refine $\mathbf{x}^{*}$ in the regions suggested by the initial stage of the global search [24-26]. While a hybrid scheme can reduce the required number of starting points and thus the number of evaluations of $f$, it necessarily introduces latency because the choice of new starting points is made after $\mathbf{x}^{*}$ has been evaluated from some previous starting points. This latency would limit the extent of parallel scaling

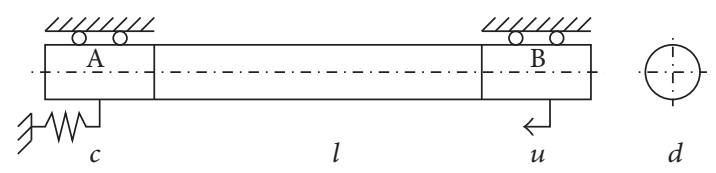

FIgURE 1: Schematic of the buckling FE model. The bar is a circular cylinder of gauge length $l$ and of diameter $d$. Side surface nodes in region $\mathrm{A}$ are attached to a spring of stiffness $c$. Prescribed axial displacement $u$ is applied to all side surface nodes in region $\mathrm{B}$.

and thus increase the time required to find the estimate of the global optimum.

Full automation of the simulation-based optimisation process, including automatic FE mesh generation, simulation and data extraction, and hierarchical parallelisation are the main novelties of this work. Also, this might be the first time that BOBYQA was applied to a real world industrial problem.

\section{The Method}

Many parameters affect the results of an FE simulation of buckling of steel reinforcing bars, the elastic tensor, the compliance of the testing machine, $c^{-1}$ (see Figure 1), initial imperfection or misalignment, the type of FE boundary conditions, and the plastic constitutive model. Not all parameters affect the results in equal measure. Although the compliance of the testing machine affects the displacements at all stages in the simulation, it is assumed that the plastic hardening model will dominate the onset of yield and postbuckling behaviour.

Assuming isotropic elasticity, the elastic behaviour is dominated by Young's modulus. Accordingly it is possible to fit the elastic and the plastic parameters separately. Critically the elastic properties and the test machine compliance are subject to linear optimisation and hence are much easier to fit. This subproblem is not addressed here, but the results are $E=$ $210 \mathrm{GPa}$ and $c=630 \mathrm{kN} / \mathrm{mm}$. Poisson's ratio has negligible effect on the elastic part of buckling response; hence the most often quoted value for steel, of 0.33 , was adopted for it.

Isotropic power hardening is assumed here for the plastic constitutive model:

$$
q=\sigma_{0}+k \epsilon_{q}^{n}
$$

where $q$ is the von Mises flow stress, $\epsilon_{q}$ is the equivalent strain, $\sigma_{0}$ is the initial yield stress, $k$ is the hardening modulus, and $n$ is the hardening exponent.

The FE simulation of buckling is conducted in two stages. In the first stage the first principal mode is calculated via the solution of the eigenvalue/eigenvector problem. In the second stage the geometry of the model is perturbed by the first principal mode multiplied by a small factor. A schematic of the FE model is shown in Figure 1. All side surface nodes in region $\mathrm{A}$ of the mesh are attached to a spring of stiffness $c$ along the axis of the cylinder. This was done to simulate experimental machine compliance, to make sure the FE predicted load/displacement traces can be compared against the experimental data. All side surface nodes in region $\mathrm{B}$ had a prescribed axial displacement $u$. These boundary conditions were chosen to simulate the grip constraints of the 
TABLE 1: Initial imperfections used in the buckling models.

\begin{tabular}{lccccc}
\hline$l / d$ & 5 & 8 & 10 & 15 & 20 \\
Imperfection, $\mathrm{mm}$ & 1.25 & 1.1 & 0.8 & 0.2 & 0.14 \\
\hline
\end{tabular}

experiment. The maximum initial imperfection amplitude for each model is given in Table 1.

Although it is possible and desirable to include the imperfection factor in the list of parameters to optimise, this was not done in this work for the following reasons. (1) Higher values of imperfection are required in the shorter FE models to induce the buckling mode of deformation, and lower values are required in the longer FE models to reach the peak load observed in experiments. It is not clear whether different values of initial imperfections are consistent with the experiments. (2) Allowing for different imperfection in each of the five FE models would add a further five parameters to optimise and make global optimisation run times prohibitively high. More details on run times are given in Sections 3 and 4.

FE analysis of buckling calculates load/displacement data. The objective function, $f$, was constructed as

$$
f(\mathbf{x})=\frac{1}{M} \sum_{m=1}^{M} \frac{1}{P_{m}^{\mathrm{FE}}} \sum_{i=1}^{P_{m}^{\mathrm{FE}}}\left(L_{i m}^{\mathrm{FE}}-L_{i m}^{\mathrm{exp}}\right)^{2}
$$

where $\mathbf{x}$ is the vector of plastic properties: $\mathbf{x}=\left(\sigma_{0}, k, n \times 10^{3}\right)$ (the factor of $10^{3}$ is used to ensure good scaling); $P_{m}^{\mathrm{FE}}$ is the number of load/displacement data points calculated by $m$ th FE model; $L_{i m}^{\mathrm{FE}}$ and $L_{i m}^{\text {exp }}$ are the FE and the experimental loads for $i$ th displacement data point from $m$ th model and $m$ th experiment; $M$ models are used in total. Double scaling, over the number of the data points in each FE model, $P_{m}^{\mathrm{FE}}$, and over the number of the FE models, $M$, ensures that the objective function is not sensitive to changing these parameters.

The mechanical meaning of $f$, as defined by (2), is the square of the load residual per point.

Each experiment provided roughly 4,000 load/displacement data points. Implicit $\mathrm{FE}$ solver required $P_{m}^{\mathrm{FE}}=15$ to $P_{m}^{\mathrm{FE}}=25$ load steps for the complete deformation paths in buckling models. Therefore, only about $1 \%$ of available experimental data was used in $f$. Linear interpolation of experimental data was used if no exact experimental displacement matching the FE value was available. Linear interpolation was accepted because of relatively very high sampling rate in the experiments.

In this work equal importance is given to all $\mathrm{FE}$ data points. However, it is possible, in future, to add weights to (2), for example, to give more importance to data at the onset of buckling, or to postbuckling behaviour [27].

The problem is cast in the standard constrained local minimisation form:

$$
\text { find } \begin{aligned}
\mathbf{x}^{*} \mid f\left(\mathbf{x}^{*}\right) & =\min _{\mathbf{x}} f(\mathbf{x}) \\
a_{i} & \leq x_{i} \leq b_{i}, \quad i=1, \ldots, m, \mathbf{x}, \mathbf{a}, \mathbf{b} \in \mathscr{R}^{m},
\end{aligned}
$$
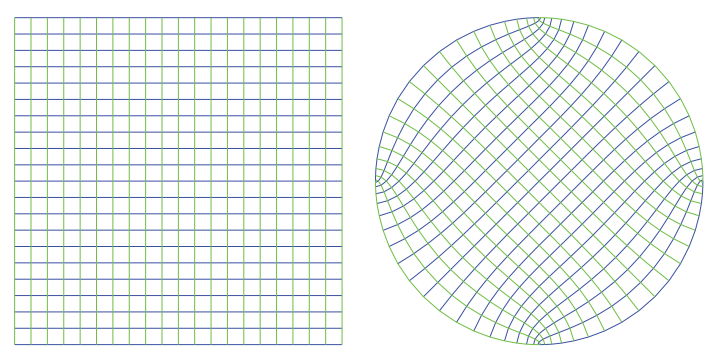

Figure 2: Conformal mapping of a square onto a circle, used for automatic parametric mesh generation.

where $m=3$ and $\mathbf{a}$ and $\mathbf{b}$, are respectively, the lower and the upper bounds for $\mathbf{x}$.

This minimisation problem is a nonlinear least squares problem where $f$ is supplied by the postprocessing of the $\mathrm{FE}$ analysis. No prior knowledge of $f$ is available. In particular, analytical derivatives of $f$ are not available. However, in this work $f$ is expected to be at least piecewise smooth, with derivatives of approximated $f$ providing useful information.

BOBYQA [28] package was used in this work for local optimisation. BOBYQA stands for bound optimisation by quadratic approximation. It does not require derivatives of $f$. The method is based on iterative fitting of a quadratic surface over trial points. The choice of the trial points is based on the trust region and on the conjugate gradient method [28]. BOBYQA was chosen for this work because (1) it offers a good overall performance for a range of optimisation problems [29,30], with particular strengths in refining a near optimal solution and solving problems with large numbers of variables, for example, over 30 [31], (2) it is easy to use [32], (3) it is written in standard portable Fortran, and (4) it is distributed under GPLv2 license, allowing user modification.

The Abaqus 6.14 FE package was used in this work. Firstorder hexahedra elements were used throughout. Parametric, script driven mesh generation is important to ensure a fully automatic FE solution pipeline, including pre- and postprocessing. First a unit square is meshed with a regular square grid. Then the unit square is extruded in the third direction for a required length to generate 1st-order hexahedra. Finally each unit square is mapped conformally onto a circle of arbitrary radius to create a circular cylinder (Figure 2). This approach is preferred in this work because it is fully automatic for arbitrary $l$ and $d$, which makes it optimal for simulating experiments with different $l / d$ ratios. Conformal mapping ensures no mesh distortion for any mesh density. The Fortran code implementing conformal mapping was adopted from the Matlab code by Fong [33, 34]. All our code used for this work is freely available online at http://optpack.sf.net under 2 -clause BSD license. Figure 3 shows the FE meshes used in the buckling analysis. Prior to the application of conformal mapping all finite elements are cubes with edge lengths of $1 \mathrm{~mm}$. In the final mesh most elements are of similar size, except for four clusters of small elements, about $0.1 \mathrm{~mm}$ to $0.5 \mathrm{~mm}$; see Figures 2 and 3 . 


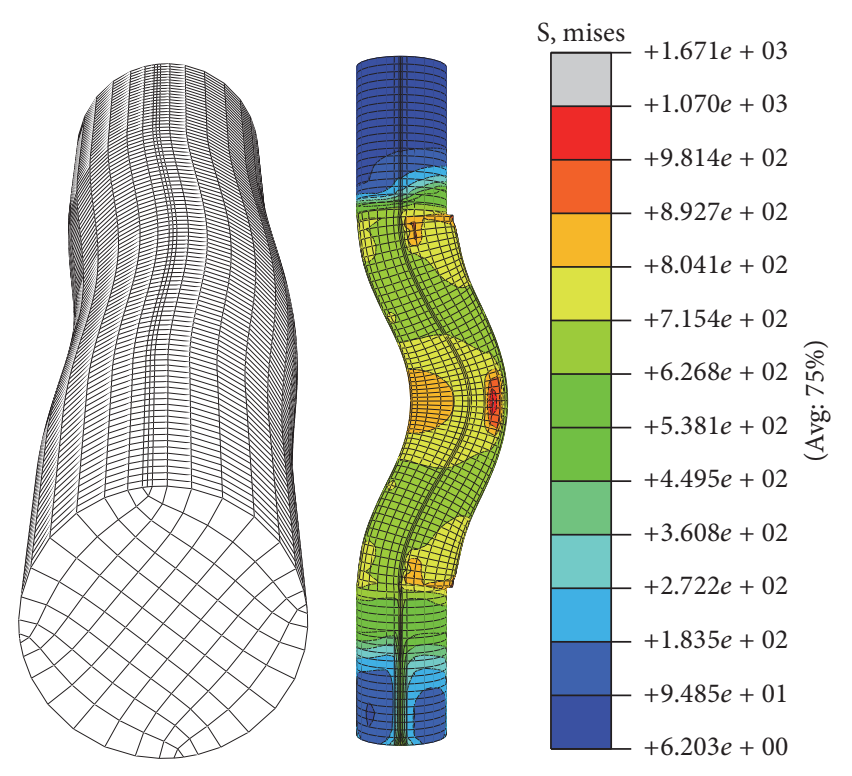

FIGURE 3: FE meshes of the $l / d=5$ specimen, showing on the left the buckling mesh before the application of the loading, with initial imperfection in the form of the 1st mode, calculated by the eigenvector/value analysis. The maximum deflection is $1 \mathrm{~mm}$. On the right is the contour plot of the von Mises stress at $10 \mathrm{~mm}$ axial displacement. Note highly distorted elements in the regions adjacent to the constraints. Also note that the location of the maximum stress is on the side surface, closer to the outer region with positive curvature, while at the same time the stress is higher at the saddle point (inner) than at the outer point, with the maximum deflection. This fact has important implications for postbuckling fatigue and fracture behaviour of reinforcing bars $[1,2,5]$.

\section{Local Optimisation and Parallelisation}

The complete process for evaluating $\mathbf{x}^{*}$ from each starting point on a Linux cluster is as follows. Five FE models are generated with $l / d=5,8,10,15,20$ to match the experiments. Eigenvalue/vector analyses are performed and the first principal modes (deformed shapes) are saved to file. Note that the eigenvalue/vector analysis does not depend on the parameters which are being optimised. Hence these analyses need to be run only once. The portable batch system (PBS) is used to set up the parallel environment, reserve the computational nodes for the job, and launch the main optimisation executable. This executable program calls subroutine BOBYQA. BOBYQA sets trial values for $\mathbf{x}$ and calls subroutine CALFUN. CALFUN prepares the Abaqus user hardening subroutine UHARD [35] from $\mathbf{x}$ and launches a shell script that concurrently runs five Abaqus FE models of buckling. Each Abaqus job is executed in parallel using MPI. The shell script waits for all FE jobs to complete and extracts the load/displacement data from each model. The load/displacement data is passed back to CALFUN, which calculates $f$ and passes it back to BOBYQA. BOBYQA algorithm then updates the quadratic approximated surface and the size of the trust region and calculates the direction and length of the next step and the next trial $\mathbf{x}$. This process is continued to convergence. It is illustrated in Figure 4.
The behaviour of $f$, (2), is not known. It might have multiple local minima, change very rapidly or very slowly in one or more of its arguments, or even be discontinuous. Indeed, it was observed that, at least in the most slender model, $l / d=20$, with very small initial imperfection, $0.14 \mathrm{~mm}$ (see Table 1), higher buckling modes can be induced for particular $\mathbf{x}$ (see Figure 5). These models, of course, predict much higher loads than the models which buckle in the first mode. Although this phenomenon is interesting in itself, its study is left for the future. For the purposes of this work the important consequence is that $f$ is likely to be discontinuous near such $\mathbf{x}$.

These factors present a number of complications to a numerical optimisation technique. First, the shape of the approximated function might change dramatically with each new function evaluation. This will lead to a rapid change of the trust region size and the search direction. This might result in poor convergence or inability to converge. Second, the process might converge to different local minima depending on the starting point. This problem exists even for functions with known derivatives; however it is much more acute when derivatives are not known.

The key input parameters to BOBYQA are the starting point, $\mathbf{x}_{0}$, the starting and the final trust region radii, $\rho_{0}$ and $\rho_{f}, \rho_{0}>\rho_{f}$, and the bounds $\mathbf{a}$ and $\mathbf{b}$; see (3).

BOBYQA constructs the starting approximated surface by taking points with distance $\rho_{0}$ from $\mathbf{x}_{0}$. As shown in Table 2, $\rho_{0}$ has a significant effect on the required number of evaluations of $f$ and on $\mathbf{x}^{*}$. In this work values between $\rho_{0}=100$ and $\rho_{0}=10$ were tried.

BOBYQA terminates the search when the trust region radius is reduced to $\rho_{f}$. Powell suggests that the local minimum is within $10 \rho_{f}$ from its last estimate of $\mathbf{x}^{*}$ [28]. In this work the accuracy of $5 \mathrm{MPa}$ is considered adequate for $\sigma_{0}$ and for $k$. Hence $\rho_{f}=0.5$ was used. Note that before passing to BOBYQA $n$ was scaled by a factor of $10^{3}$ to make all components of $\mathbf{x}$ of the same order. Therefore the accuracy of $n$ is $5 \times 10^{-3}$, which is considered adequate. The relatively small difference between $\rho_{0}$ and $\rho_{f}$, in the order of $10^{2}-10^{3}$, leads to very rapid convergence, which is desirable because each evaluation of $f$ is expensive.

An important factor in this work that tight bounds on each parameter were available from prior work on this steel [3-5], where these parameters were estimated using ad hoc trial and error procedures, common in practical engineering. The following bound vectors were deemed reasonable: $\mathbf{a}=$ $(400,300,250)$ and $\mathbf{b}=(600,500,450)$, meaning that each variable was constrained as $\sigma_{0}=x_{1} \in[400,600], k=x_{2} \in$ $[300,500]$, and $n=10^{-3} \times x_{3} \in[0.25,0.45]$.

The influence of varying the starting point, $\mathbf{x}_{0}$, on the rate of convergence and on $\mathbf{x}^{*}$ was also investigated. The results are shown in Table 2.

The results show that $f$ is shallow around the local minima; see Figure 6. The first $2 m+1=7$ evaluations of $f$ are done on points $\mathbf{x}_{0}$ with each component of this vector perturbed by $\pm \rho_{0}$ or on the bounds $\mathbf{a}$ and $\mathbf{b}$ if $\mathbf{x}_{0} \pm \rho_{0}$ is outside of the bounds. These evaluations are used to construct the starting approximation to $f[28]$. Therefore no convergence 


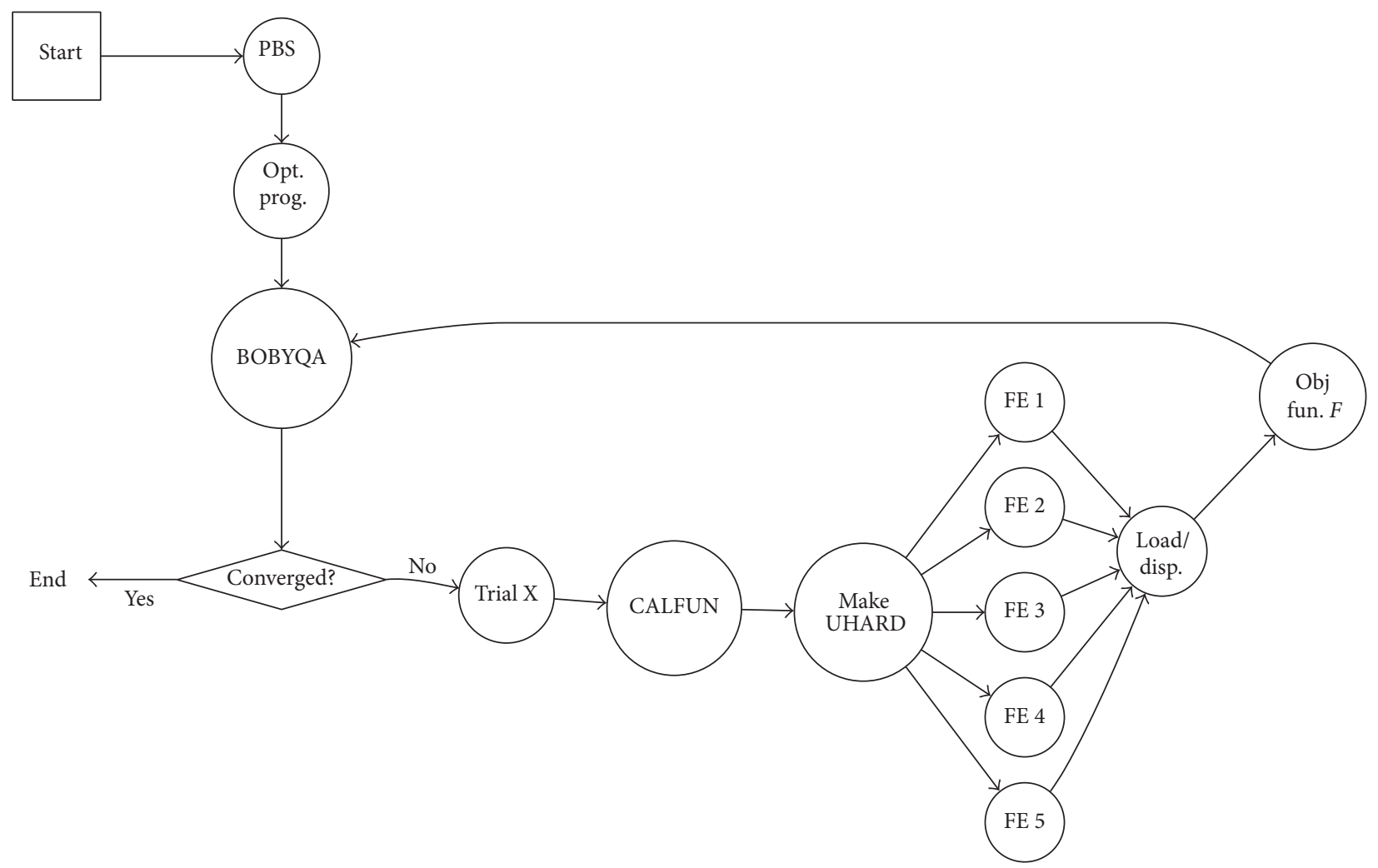

FIGURE 4: Schematic diagram of the optimisation work flow showing the order of execution and the main iteration loop. The five concurrent FE calculations account for over $99 \%$ of the total run time.

is expected in the first 7 iterations. From iteration 8 onward convergence is very rapid and little improvement is achieved beyond iteration 15 or in some cases as early as iteration 11; see Figure 7. This means that quadratic approximation used in BOBYQA is well suited for $f$ around the local minima. This fact and the use of relatively high $\rho_{f}$ ensure that few evaluations of $f$ are required to find $\mathbf{x}^{*}$.

The local minima are sensitive to $\mathbf{x}_{0}$ and $\rho_{0}$; see Table 2 and Figure 7. If $\mathbf{x}_{0}$ is close to $\mathbf{x}^{*}$, then using smaller $\rho_{0}$ can produce a better optimum, as evidenced by cases $1-3$. If no good guess for $\mathbf{x}_{0}$ is available, high $\rho_{0}$ is advisable. Taking too low $\rho_{0}$ might result in premature convergence, as in case 7 . Cases 4 , 6 , and 8 show that $\rho_{0}=100$ is sufficient for converging to a good optimum, even when $\mathbf{x}_{0}$ is far from $\mathbf{x}^{*}$.

Two levels of parallelisation were exploited for local search: (1) five FE simulations were run concurrently and independently and (2) Each FE calculation was done using multiple MPI processes. There are likely further fine grained parallelism options in the FE solver, to do with matrix partitioning, and so forth. However, these were not available to us for this work because the Abaqus is closed proprietary software.

The University of Bristol BlueCrystal cluster was used in this work. Each node has two 8-core 2.6 GHz Sandy Bridge processors with $4 \mathrm{~GB}$ per core. It was found that the Abaqus FE solver does not scale well for the problems analysed in this work. Even the largest model, $l / d=20$, did not scale to the full node, 16 cores. The best performance for this model was achieved with 12 to 14 cores, with the scaling factor of 5 to 6 , and with 4-8 cores for models with lower $l / d$, with the scaling factor of 2 to 3 . Consequently the best local search performance was achieved with three computational nodes. The first node was used to analyse models with $l / d=5$ on 4 cores and with $l / d=15$ on 12 cores. The second node was used to analyse models with $l / d=8$ and $l / d=10$, each on 8 cores. The model with $l / d=20$ was analysed on the third node with 14 cores. Each evaluation of $f$ took on average $2.6 \mathrm{~min}$ or 23 evaluations per hour. According to Table 2 this means that each starting point can be evaluated in 1 to 1.5 hours.

\section{Global Optimisation and Parallelism}

In addition to the two levels of parallelism exploited for local search, in global multistart search the third level was exploited too: (3) multiple starting points were evaluated concurrently and independently.

In principle any and all parallelism options can be enabled; however for optimal performance, these have to be chosen wisely, given hardware, software, and licensing limitations. The PBS system limits the total execution time and the number of nodes available to a job. The Abaqus FE requires extra license tokens for each core used for parallel execution. The total number of license tokens available to us is limited. 


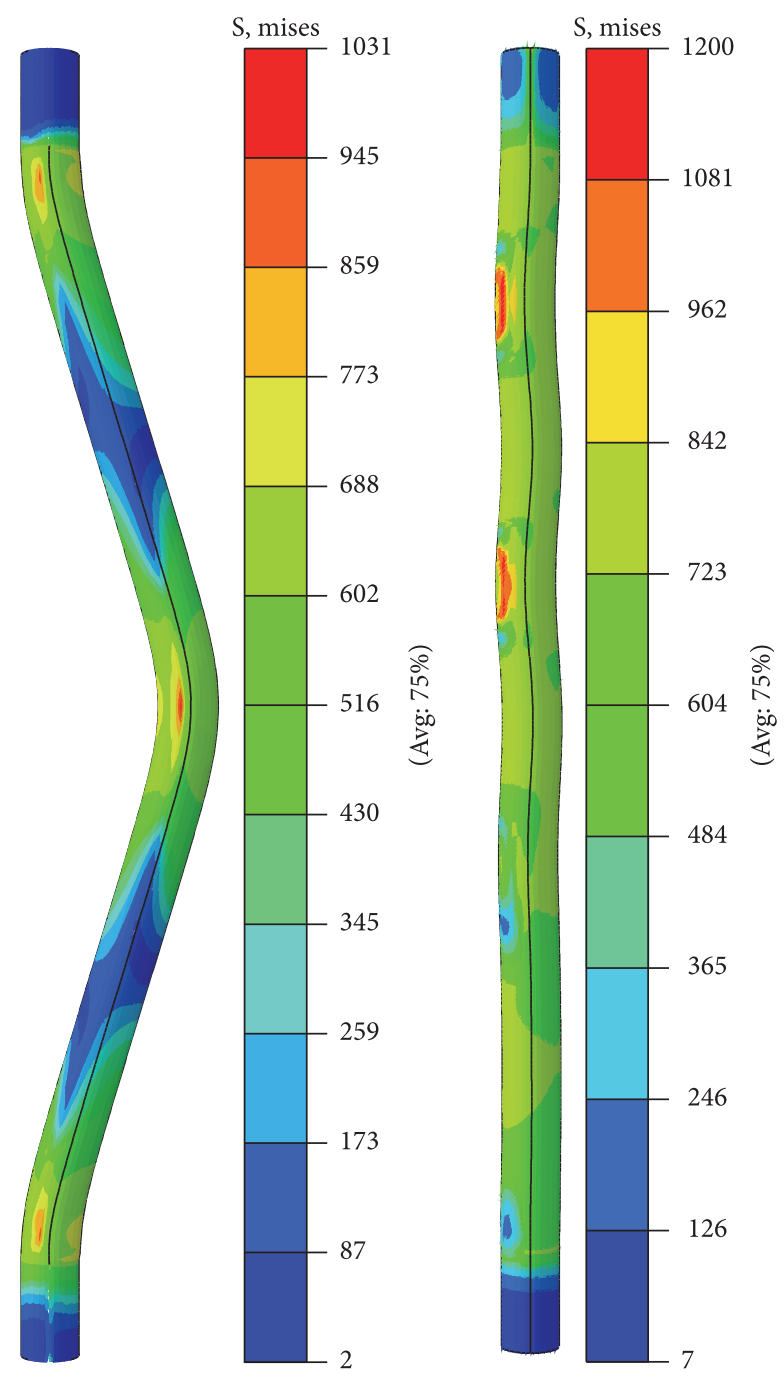

FIgURE 5: Buckled shapes of the $l / d=20$ bar with initial imperfection of $0.14 \mathrm{~mm}$ (see Table 1), from Case 1, showing, on the left, the bar with plastic properties of $\mathbf{x}^{*}: \sigma_{0}=493.0 \mathrm{MPa}$, $k=413.0 \mathrm{MPa}$, and $n=0.3303$, buckled in the 1st mode, $f=2.666$, and, on the right, the bar with plastic properties of 2 nd evaluation of $f: \sigma_{0}=600.0 \mathrm{MPa}, k=400.0 \mathrm{MPa}$, and $n=0.3500$, buckled in a higher mode, $f=6.1 \times 10^{2}$. See Figure 6 and Table 2 for more details. The contour plots show Mises stress in MPa.

These factors lead to several parallelisation strategies. At one extreme is the approach in which each FE job is executed serially, thus allowing for a maximum number of concurrent FE jobs to be run. In this case relatively many starting points can be evaluated concurrently, but slowly. At the other extreme is execution of each FE job with maximum parallelisation, using all available Abaqus license tokens and all available PBS nodes. In this mode starting points are evaluated serially, but fast. As mentioned in the previous section, the Abaqus scaling is limited. Hence, after some experimentation, it was concluded that optimum FE parallel performance is achieved when 3 nodes are used to evaluate each starting point. Four starting points, chosen at random, were evaluated concurrently, requiring 12 nodes (196 cores)

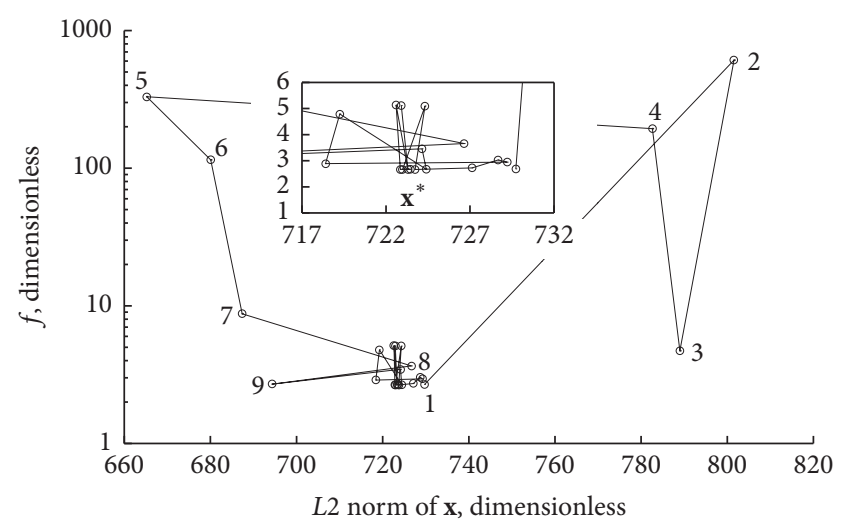

Figure 6: Minimum search path for case 4. The numbers indicate the order in which $f$ was evaluated. An inset shows the behaviour of $f$ around $\mathbf{x}^{*}$, evaluations of $f$ from 10 to 24 . See Table 2 for details of the cases.

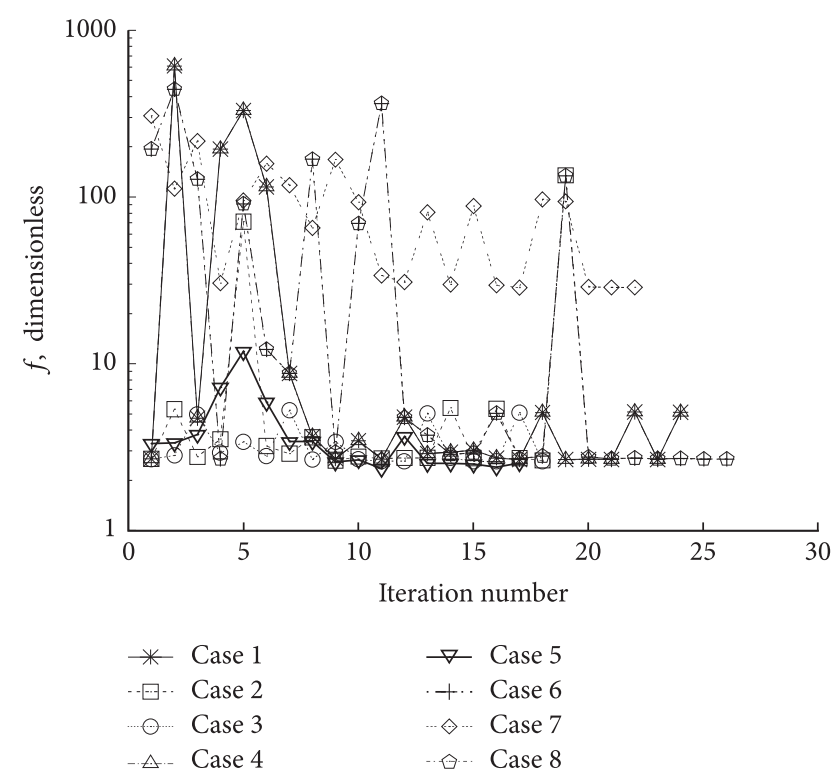

Figure 7: Rate of convergence for cases detailed in Table 2.

and 2,232 Abaqus license tokens. Availability of the Abaqus license tokens was the ultimate limitation. With this level of concurrency 3 starting points were evaluated per hour. A total of 50 points have been evaluated and Table 3 shows five lowest minima found.

\section{Validation}

Figure 8 compares the experimental load/displacement traces with those predicted by the FE with the lowest minimum plastic properties, line 1 of Table 3 . To judge whether $f\left(\mathbf{x}^{*}\right) \approx$ 2.5 is a good fit or not, one would have to compare the residual between the model and experiments with that between repeated experiments. However, repeated experimental data was not available for this work.

Instead $\mathbf{x}^{*}$ is validated on a different experiment. In this work a tensile test was used. Figure 9 shows a good match 
TABLE 2: Influence of the starting point and the initial trust region size on the rate of convergence and the local minima. $n=10^{-3} \times x_{3}^{*}$.

\begin{tabular}{|c|c|c|c|c|c|}
\hline Case & $\rho_{0}$ & $\mathbf{x}_{0}$ & $f$ eval. & $\mathbf{x}^{*}$ & $f\left(\mathbf{x}^{*}\right)$ \\
\hline 1 & 100 & $(500,400,350)$ & 24 & $(493.0,413.0,330.3)$ & 2.666 \\
\hline 2 & 30 & $(500,400,350)$ & 19 & $(507.6,387.2,348.5)$ & 2.621 \\
\hline 3 & 10 & $(500,400,350)$ & 18 & $(505.0,396.9,353.1)$ & 2.593 \\
\hline 4 & 100 & $(590,490,440)$ & 24 & $(493.0,413.0,330.3)$ & 2.666 \\
\hline 5 & 30 & $(410,480,260)$ & 17 & $(433.9,478.0,266.4)$ & 2.351 \\
\hline 6 & 100 & $(410,480,260)$ & 26 & $(500.0,400.0,349.5)$ & 2.681 \\
\hline 7 & 50 & $(401,301,400)$ & 22 & $(452.1,354.9,448.2)$ & 28.610 \\
\hline 8 & 100 & $(401,301,400)$ & 26 & $(500.0,400.0,349.5)$ & 2.681 \\
\hline
\end{tabular}

TABLE 3: Five lowest minima for multistart global optimisation. Columns 2 and 3 show the minima from using only buckling experimental data, Section 4. Columns 4 and 5 show the minima from using buckling and tensile experimental data, Section 6.

\begin{tabular}{llrrr}
\hline & \multicolumn{2}{c}{} & \multicolumn{2}{c}{ Data for Section 6 } \\
& $f\left(\mathbf{x}^{*}\right)$ & Data for Section 4 & $f\left(\mathbf{x}^{*}\right)$ & $(434.2,394.9,202.1)$ \\
\hline 1 & 2.534 & $(451.6,475.5,290.1)$ & 2.355 & $(436.5,397.8,209.1)$ \\
3 & 2.564 & $(451.0,484.6,297.8)$ & 2.363 & $(428.2,414.7,200.7)$ \\
4 & 2.570 & $(450.3,373.0,210.9)$ & 2.369 & $(451.0,473.9,290.2)$ \\
5 & 2.575 & $(442.5,482.2,281.4)$ & 2.576 & $(506.6,446.0,393.0)$ \\
\hline
\end{tabular}

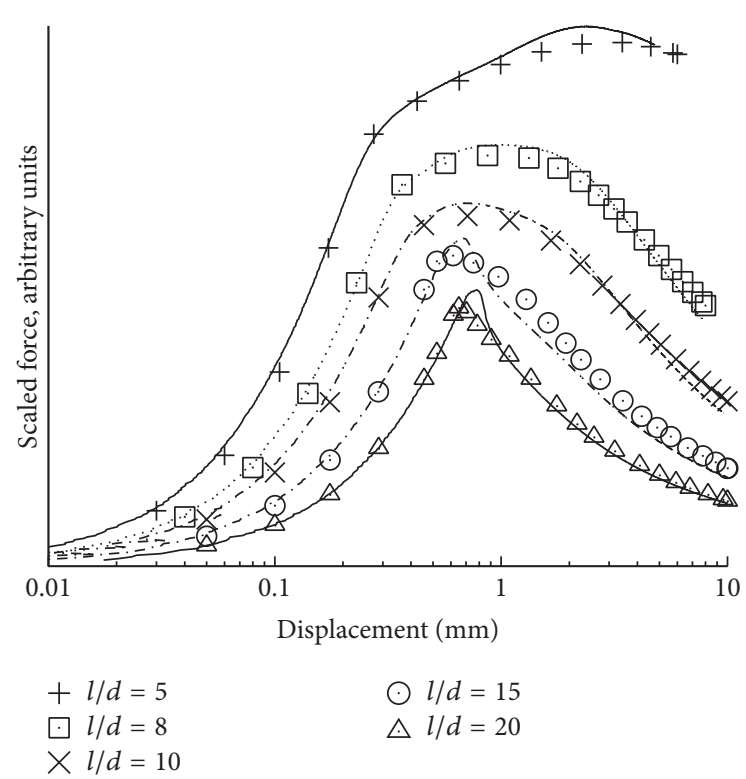

Figure 8: FE load-displacement traces obtained with the best estimate of the global minimum using only the buckling data, $\sigma_{0}=$ $451.6 \mathrm{MPa}, k=475.5 \mathrm{MPa}, n=0.2901$, and $f\left(\mathbf{x}^{*}\right)=2.534$, are shown as symbols. Superimposed lines show the experimental data. Note that the curves are deliberately separated for clarity, via arbitrary scaling of the load for each dataset. The plot is used exclusively to highlight a match between the FE and the experiment for each geometry.

between the experimental load/displacement curve and those predicted by the FE with the fitted plastic properties from Table 3. However, none of the five minima describe the last stages of the deformation well (see inset in Figure 9),

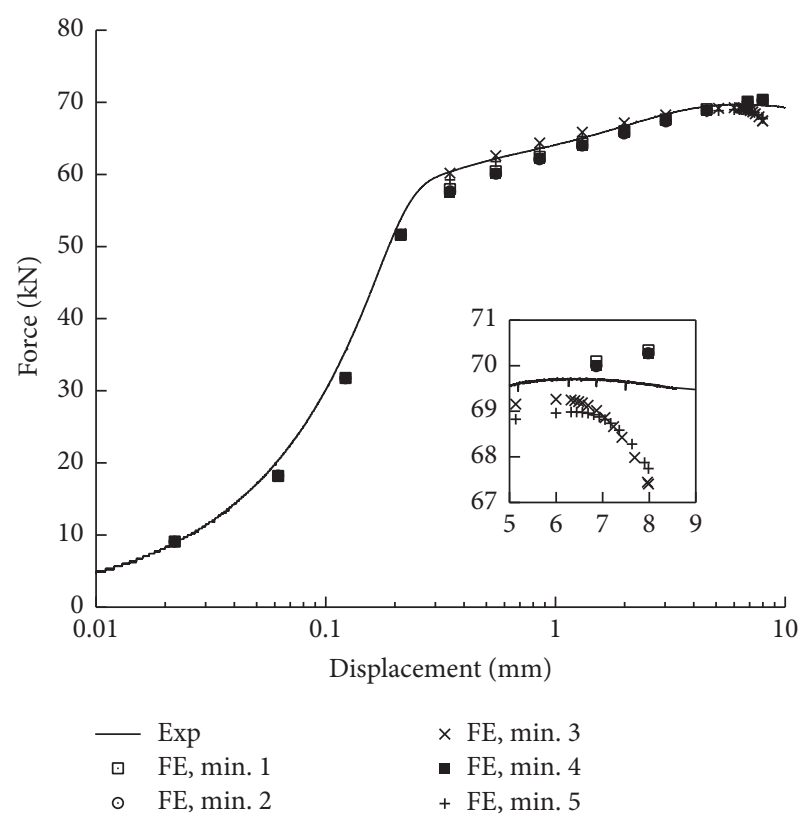

FIGURE 9: FE load/displacement traces calculated with the hardening properties of Table 3 are shown with symbols. The solid line shows the experimental data.

where necking is accompanied by ductile damage. This is the limitation of the 3-parameter plastic hardening model adopted in this work.

Note that the validation step forms an integral part of the autotuning method. This means that some distinct data must be deliberately left for validation and not included into the formulation of $f,(2)$. 


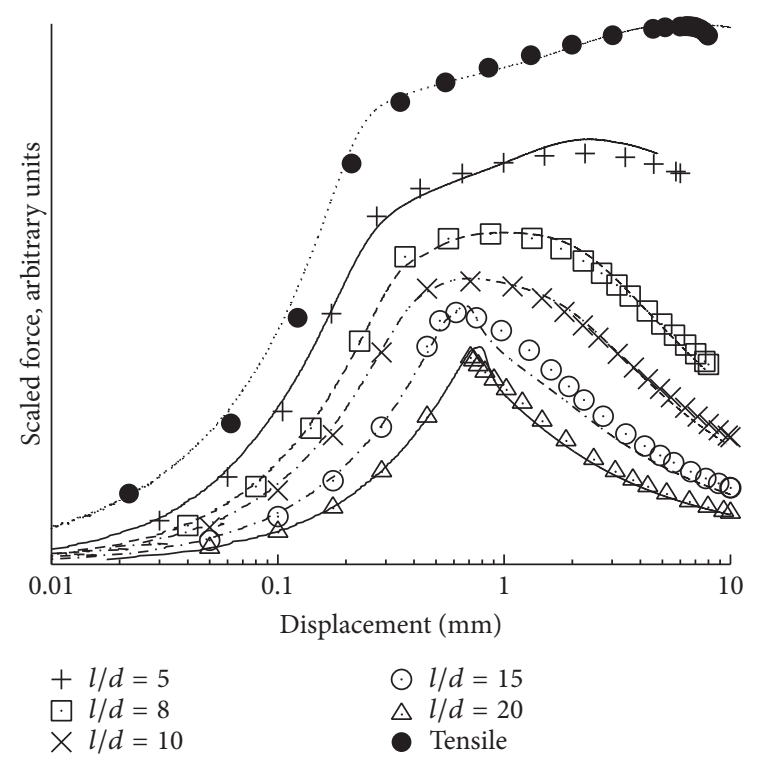

Figure 10: FE load-displacement traces obtained with the best buckling and tensile estimates of the global optimum, $\sigma_{0}=$ $434.2 \mathrm{MPa}, k=394.9 \mathrm{MPa}, n=0.2021$, and $f\left(\mathbf{x}^{*}\right)=2.355$ (see Table 3), are shown as symbols. Superimposed lines show the experimental data. Note that the curves are deliberately separated for clarity, via arbitrary scaling of the load for each dataset. The plot is used exclusively to highlight a match between the FE and the experiment for each geometry.

\section{Global Optimisation with No Validation}

While the validation gives the user some measure of the goodness of fit, it requires that some experimental data is left out of the optimisation. As a result the optimisation is inevitably done over a smaller range of stress-strain histories, thus reducing the range of applicability of the tuned plastic constitutive model.

One can use all available experimental data for optimisation, at the expense of losing the measure of goodness of fit. However, the fitted plastic model will have a wider applicability.

If the tensile test data is included into $f,(2)$, then different local minima are obtained. The five lowest minima obtained from 25 starting points are shown in Table 3. Figure 10 shows the experimental and the FE load/displacement traces at the best discovered estimate of the global optimum.

It is interesting to note that minima $1-3$ and 5 found using both the buckling and the tensile data in $f$ are distinctly different from the lowest minima found using only the buckling data. However, the 4th lowest minima are quite similar in both cases. It is hard to draw definitive conclusions from these observations because relatively a small number of starting points, 50 and 25, respectively, have been tried. Given enough time, it is possible that even lower minima might be discovered in both cases.

\section{Discussion and Further Work}

The main discovery of this work is that no single clear estimate of the global optimum has emerged. The five lowest local minima discovered when using only the buckling data or when using both the buckling and the tensile data have very different $\mathbf{x}^{*}$ with very similar $f\left(\mathbf{x}^{*}\right)$. Although it is logical to accept $\mathbf{x}^{*}$ with the lowest $f\left(\mathbf{x}^{*}\right)$ as the best estimate of the global optimum, the confidence of this choice would be low.

Section 6 shows that adding more data into the optimisation results in only a very small improvement in the prominence of the estimate of the global optimum. However, the best discovered estimate of the global optimum with the tensile data included is quite different from that obtained with just the buckling data. In other words, the discovered optimum might be sensitive to the noise in experimental data.

One possible explanation of this is that diversity of stress/strain histories in the data cannot be fully represented by the 3-parameter isotropic power hardening model. The mechanical explanation of this is that the isotropic power hardening might not adequately represent the mechanical behaviour of structural steel, particularly at large strain, where some ductile damage mechanism might be activated. It is therefore suggested to explore in future a combined isotropic/kinematic hardening model, as the model regions close to the constraints, and those with the maximum off-axis deflection, experience reverse plasticity, first compression, followed by tension at large buckling deformations. In addition it is suggested to explore fitting of an anisotropic hardening model, such as Hill's orthotropic model, which previously was fitted successfully with the virtual fields method [13]. Depending on the manufacturing process, reinforcing bars might have texture and hence be significantly anisotropic.

It would be interesting to explore whether the addition of the initial imperfection to the optimisation parameters would result in a better estimate for the global optimum. This work requires a substantial increase in the number of optimisation parameters, 5 in the present case. Therefore it is deemed feasible only when better scalability of the FE solver has been achieved.

Scalable and scriptable FE solver is required to reduce calculation time of $f$. If each evaluation of $f$ can be done in less than a minute, then it will become feasible to add more variables to $\mathbf{x}$, such as the initial imperfection. When $f$ can be evaluated in just a few seconds, then optimisation of more complex plastic and/or damage models will become feasible, for example, combined kinematic/isotropic hardening or the Gurson-Tvergaard-Needleman continuous damage model [36]. The use of a damage model will also help better describe the necking behaviour in the tensile tests, something that a 3-parameter hardening model cannot do, as described in Section 5. It is likely that this will require FE codes scalable up to tens or hundreds of thousands of cores. One possible candidate is ParaFEM, which showed excellent scaling on HECToR and ARCHER, the UK national supercomputers, that is, Tier-1 systems [37]. With exascale era widely expected to start by about 2020 [38], this work is a small step towards enabling exascale computing for practical mechanical or civil engineering applications. 


\section{Conclusions}

Multistart global optimisation, with BOBYQA used for local search, has proved successful for autotuning a 3-parameter isotropic power hardening model, where the objective function was a scaled sum of the squared differences between the experimental and the FE load/displacement data for elastoplastic buckling of steel rods. Thus the optimisation was a nonlinear least squares problem.

Convergence of BOBYQA is sensitive to the starting trust region radius, $\rho_{0}$. For small $\rho_{0}$ BOBYQA can converge prematurely, because the objective function is shallow around the minima. The use of a large initial trust region radius is thus recommended.

Higher mode buckling was predicted by FE for specific combinations of the model parameters, resulting in very high gradients or even discontinuities of the objective function.

The quality of the best discovered global optimum was assessed using a separate, post-optimisation validation step.

However, poor prominence of the best discovered global optimum raises further questions, which the authors intend to address in future work: (1) How do the type and the amount of experimental data affect the design of the objective function? (2) If some knowledge of the influence of the model parameters on the stress/strain response is available, how can it be included into the objective function? (3) What is the type of the desired new experimental data, given a prior estimation of the global optimum?

\section{Disclosure}

Mohammad M. Kashani is now at Faculty of Engineering and Environment, University of Southampton, Southampton SO17 1BJ, UK.

\section{Competing Interests}

The authors declare that there is no conflict of interest regarding the publication of this paper.

\section{Acknowledgments}

This work was carried out using the computational facilities of the Advanced Computing Research Centre, The University of Bristol: https://www.bris.ac.uk/acrc/. Professor M. J. D. Powell died in 2015. In readme for BOBYQA package he writes: "I hope that the time and effort I have spent on developing the package will be helpful to much research and to many applications." It has been and it will.

\section{References}

[1] M. M. Kashani, A. J. Crewe, and N. A. Alexander, "Nonlinear stress-strain behaviour of corrosion-damaged reinforcing bars including inelastic buckling," Engineering Structures, vol. 48, pp. 417-429, 2013.

[2] M. M. Kashani, A. J. Crewe, and N. A. Alexander, "Nonlinear cyclic response of corrosion-damaged reinforcing bars with the effect of buckling," Construction and Building Materials, vol. 41, pp. 388-400, 2013.

[3] M. M. Kashani, L. N. Lowes, A. J. Crewe, and N. A. Alexander, "Finite element investigation of the influence of corrosion pattern on inelastic buckling and cyclic response of corroded reinforcing bars," Engineering Structures, vol. 75, pp. 113-125, 2014.

[4] M. M. Kashani, Seismic performance of corroded rc bridge piers: development of a multi-mechanical nonlinear fibre beam-column model [Ph.D. thesis], University of Bristol, Bristol, UK, 2014.

[5] M. M. Kashani, A. K. Barmi, and V. S. Malinova, "Influence of inelastic buckling on low-cycle fatigue degradation of reinforcing bars," Construction and Building Materials, vol. 94, pp. 644655, 2015.

[6] M. Berry and M. O. Eberhard, "Performance models for flexural damage in reinforced concrete columns," Tech. Rep. 162, Pacific Earthquake Engineering Research Centre, University of California Berkeley, Berkeley, Calif, USA, 2003.

[7] D. Lehman, J. Moehle, S. Mahin, A. Calderone, and L. Henry, "Experimental evaluation of the seismic performance of reinforced concrete bridge columns," Journal of Structural Engineering, vol. 130, no. 6, pp. 869-879, 2004.

[8] M. P. Berry and M. O. Eberhard, "Practical performance model for bar buckling," Journal of Structural Engineering, vol. 131, no. 7, pp. 1060-1070, 2005.

[9] A. R. Conn, K. Scheinberg, and P. L. Toint, "Recent progress in unconstrained nonlinear optimization without derivatives," Mathematical Programming, vol. 79, pp. 397-414, 1997.

[10] M. J. Powell, "On trust region methods for unconstrained minimization without derivatives," Mathematical Programming, vol. 97, no. 3, pp. 605-623, 2003.

[11] S. Avril, M. Bonnet, A.-S. Bretelle et al., "Overview of identification methods of mechanical parameters based on full-field measurements," Experimental Mechanics, vol. 48, article 381, 2008.

[12] D.-T. Nguyen, J.-G. Park, and Y.-S. Kim, "A study on yield function for Ti-6Al-4V titanium alloy sheets at elevated temperatures," Transactions of the Indian Institute of Metals, vol. 69, no. 7, pp. 1343-1350, 2016.

[13] J.-H. Kim, F. Barlat, F. Pierron, and M.-G. Lee, “Determination of anisotropic plastic constitutive parameters using the virtual fields method," Experimental Mechanics, vol. 54, no. 7, pp. 11891204, 2014.

[14] B. Banerjee, T. F. Walsh, W. Aquino, and M. Bonnet, "Large scale parameter estimation problems in frequency-domain elastodynamics using an error in constitutive equation functional," Computer Methods in Applied Mechanics and Engineering, vol. 253, pp. 60-72, 2013.

[15] J. M. Pérez Zerpa and A. Canelas, "Efficient formulations of the material identification problem using full-field measurements," Computational Mechanics, vol. 58, no. 2, pp. 235-255, 2016.

[16] V. Sundararaghavan and N. Zabaras, "A multi-length scale sensitivity analysis for the control of texture-dependent properties in deformation processing," International Journal of Plasticity, vol. 24, no. 9, pp. 1581-1605, 2008.

[17] H. Liu, S. Xu, X. Wang, J. Wu, and Y. Song, "A global optimization algorithm for simulation-based problems via the extended DIRECT scheme," Engineering Optimization, vol. 47, no. 11, pp. 1441-1458, 2015. 
[18] F. Liang, Y. Y. Guo, and R. Y. K. Fung, "Simulation-based optimization for surgery scheduling in operation theatre management using response surface method," Journal of Medical Systems, vol. 39, no. 11, article 159, 2015.

[19] S. Shan and G. G. Wang, "Survey of modeling and optimization strategies to solve high-dimensional design problems with computationally-expensive black-box functions," Structural and Multidisciplinary Optimization, vol. 41, no. 2, pp. 219241, 2010.

[20] D. R. Jones, M. Schonlau, and W. J. Welch, "Efficient global optimization of expensive black-box functions," Journal of Global Optimization, vol. 13, no. 4, pp. 455-492, 1998.

[21] R. Martí, M. G. C. Resende, and C. C. Ribeiro, "Multi-start methods for combinatorial optimization," European Journal of Operational Research, vol. 226, no. 1, pp. 1-8, 2013.

[22] Z. Ugray, L. Lasdon, J. Plummer, F. Glover, J. Kelly, and R. Marti, "Scatter search and local NLP solvers: a multistart framework for global optimization," INFORMS Journal on Computing, vol. 19, no. 3, pp. 328-340, 2007.

[23] F. V. Berghen and H. Bersini, "CONDOR, a new parallel, constrained extension of Powell's UOBYQA algorithm: experimental results and comparison with the DFO algorithm," Journal of Computational and Applied Mathematics, vol. 181, no. 1, pp. 157-175, 2005.

[24] J. Dennis and V. Torczon, "Direct search methods on parallel machines," SIAM Journal on Optimization, vol. 1, no. 4, pp. 448474, 1991.

[25] A. Tiwari, V. Tabatabaee, and J. K. Hollingsworth, "Tuning parallel applications in parallel," Parallel Computing, vol. 35, no. 8-9, pp. 475-492, 2009.

[26] M. A. Ahandani, M.-T. Vakil-Baghmisheh, and M. Talebi, "Hybridizing local search algorithms for global optimization," Computational Optimization and Applications, vol. 59, no. 3, pp. 725-748, 2014.

[27] A. Sadollah, H. Eskandar, D. G. Yoo, and J. H. Kim, "Approximate solving of nonlinear ordinary differential equations using least square weight function and metaheuristic algorithms," Engineering Applications of Artificial Intelligence, vol. 40, pp. 117132, 2015.

[28] M. J. D. Powell, “The BOBYQA algorithm for bound constrained optimization without derivatives," Tech. Rep. NA2009/ 06, Cambridge Numerical Analysis group, Department of Applied Mathematics and Theoretical Physics, Centre for Mathematical Sciences, University of Cambridge, Cambridge, UK, 2009.

[29] P. D. Conejo, E. W. Karas, and L. G. Pedroso, "A trustregion derivative-free algorithm for constrained optimization," Optimization Methods \& Software, vol. 30, no. 6, pp. 1126-1145, 2015.

[30] M. B. Arouxét, N. Echebest, and E. A. Pilotta, "Active-set strategy in Powell's method for optimization without derivatives," Computational \& Applied Mathematics, vol. 30, no. 1, pp. 171196, 2011.

[31] L. M. Rios and N. V. Sahinidis, "Derivative-free optimization: a review of algorithms and comparison of software implementations," Journal of Global Optimization, vol. 56, no. 3, pp. 12471293, 2013.

[32] S. Cheng, H. Zhan, and Z. Shu, "An innovative hybrid multiobjective particle swarm optimization with or without constraints handling," Applied Soft Computing Journal, vol. 47, pp. 370-388, 2016.
[33] C. Fong, "Analytical methods for squaring the disc," Seoul ICM 2014 poster, http://www.slideshare.net/chamb3rlain/analyticalmethods.

[34] C. Fong and B. Vogel, "Warping peirce quincuncial panoramas," in Proceedings of the 7th International Congress of Industrial and Applied Mathematics, Vancouver, Canada, 2011, http://arxiv.org/ftp/arxiv/papers/1011/1011.3189.pdf.

[35] Dassault Systèmes, Abaqus Documentation, Dassault Systèmes, Providence, RI, USA, 6.14 edition, 2015.

[36] A. Shterenlikht and N. A. Alexander, "Levenberg-Marquardt vs Powell's dogleg method for Gurson-Tvergaard-Needleman plasticity model," Computer Methods in Applied Mechanics and Engineering, vol. 237-240, pp. 1-9, 2012.

[37] I. M. Smith, D. V. Griffiths, and L. Margetts, Programming the Finite Element Method, John Wiley \& Sons, Ltd, Chichester, UK, 2015.

[38] J. Dongarra, P. Beckman, T. Moore et al., "The international exascale software project roadmap," International Journal of High Performance Computing Applications, vol. 25, no. 1, pp. 360, 2011. 


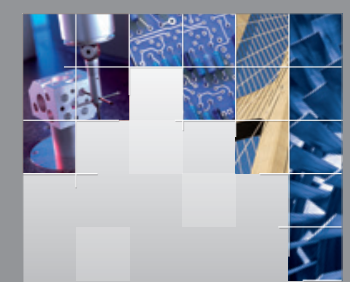

\section{Enfincering}
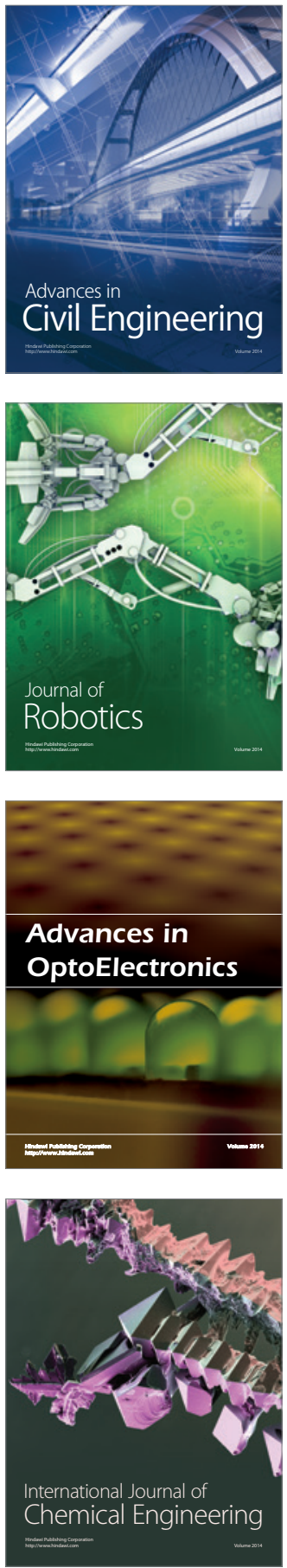

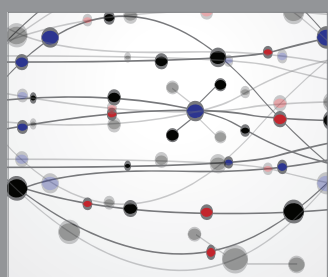

The Scientific World Journal

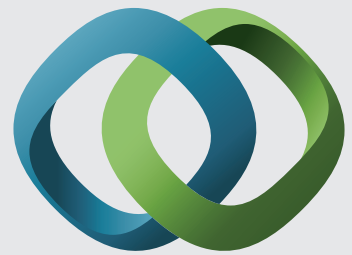

\section{Hindawi}

Submit your manuscripts at

https://www.hindawi.com
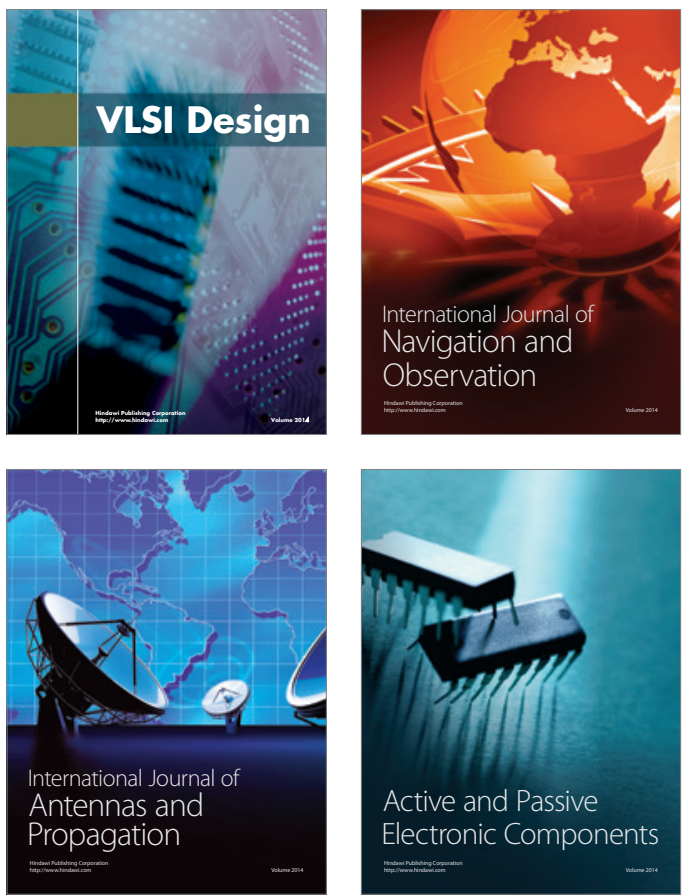
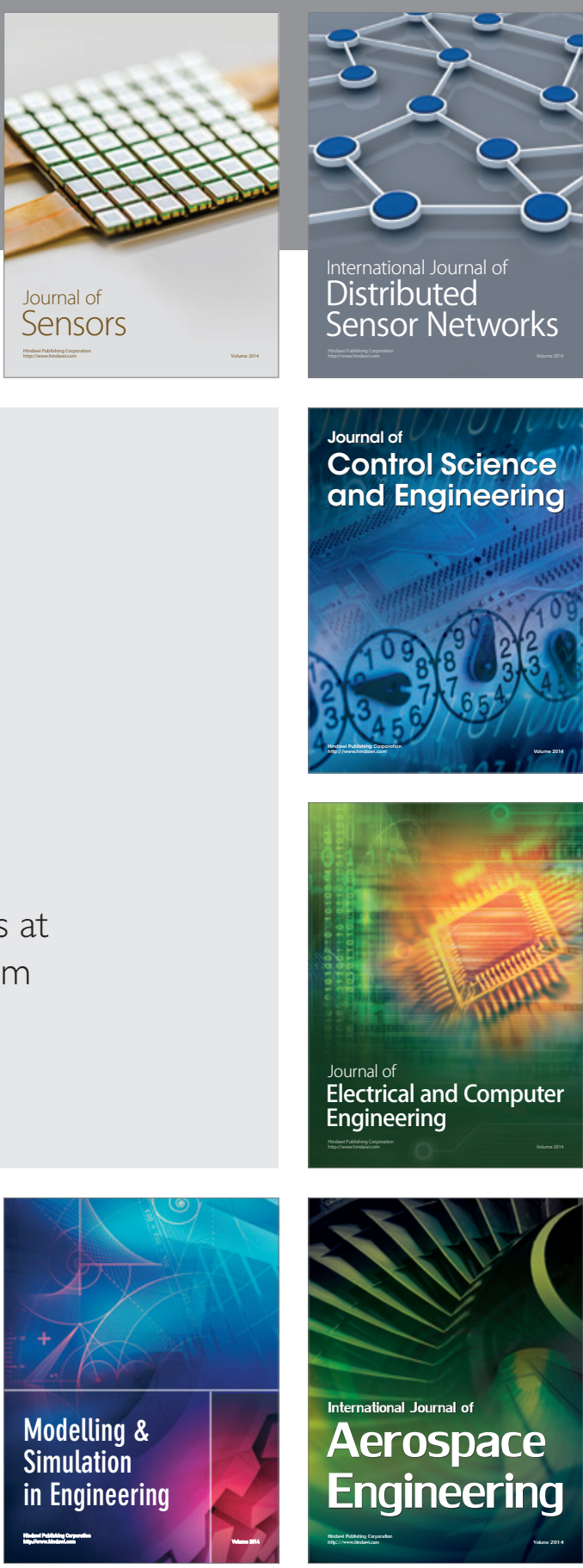

International Journal of

Distributed

Sensor Networks

$-$

Joumal of

Control Science

and Engineering
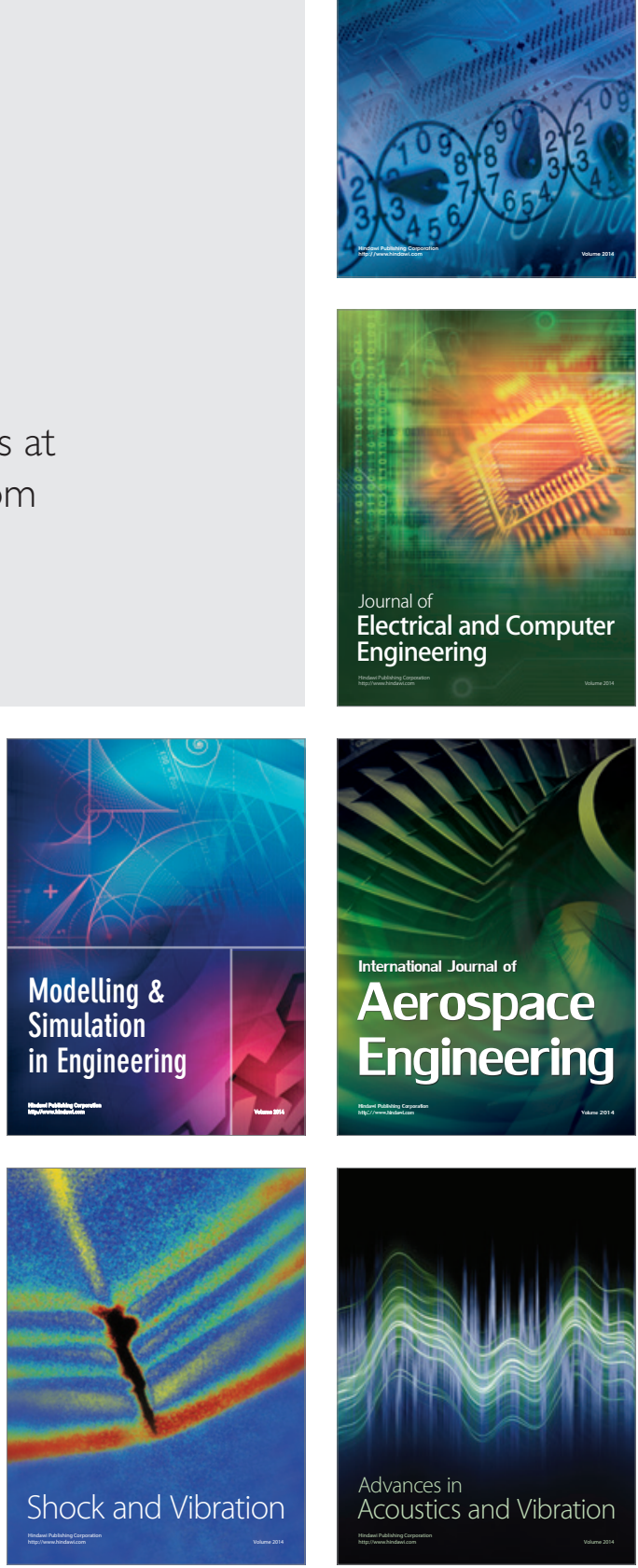